\title{
A Z-Gradient Array for Simultaneous Multi-Slice Excitation With a Single-Band RF Pulse
}

\author{
Koray Ertan (Di, ${ }^{1,2}$ Soheil Taraghinia, ${ }^{1,2}$ Alireza Sadeghi, ${ }^{1,2}$ and Ergin Atalar (D) ${ }^{1,2 *}$
}

\begin{abstract}
Purpose: Multi-slice radiofrequency (RF) pulses have higher specific absorption rates, more peak RF power, and longer pulse durations than single-slice RF pulses. Gradient field design techniques using a z-gradient array are investigated for exciting multiple slices with a single-band RF pulse.

Theory and Methods: Two different field design methods are formulated to solve for the required current values of the gradient array elements for the given slice locations. The method requirements are specified, optimization problems are formulated for the minimum current norm and an analytical solution is provided. A 9-channel z-gradient coil array driven by independent, custom-designed gradient amplifiers is used to validate the theory.

Results: Performance measures such as normalized slice thickness error, gradient strength per unit norm current, power dissipation, and maximum amplitude of the magnetic field are provided for various slice locations and numbers of slices. Two and 3 slices are excited by a single-band RF pulse in simulations and phantom experiments.

Conclusion: The possibility of multi-slice excitation with a single-band RF pulse using a z-gradient array is validated in simulations and phantom experiments. Magn Reson Med 80:400-412, 2018. (c) 2017 International Society for Magnetic Resonance in Medicine.
\end{abstract}

Key words: multi-slice excitation; simultaneous multi-slice (SMS); gradient array; RF pulse design

\section{INTRODUCTION}

In MRI, simultaneous multi-slice (SMS) imaging is an effective method of accelerating image acquisition. SMS techniques have been applied for turbo spin-echo imaging (1), echo-planar image (EPI)-based functional magnetic resonance imaging (fMRI) (2) and diffusion sequences (3). Notably, the excitation, refocusing or inversion of multiple slices requires demanding RF pulse designs. The simplest technique for designing such RF pulses is to superpose multiple single-slice RF pulses with different frequencies. However, the specific absorption rate $(\mathrm{SAR})$ and peak $\mathrm{B}_{1}$ amplitude are limiting

\footnotetext{
${ }^{1}$ National Magnetic Resonance Research Center (UMRAM), Bilkent University, Bilkent, Ankara, Turkey.

${ }^{2}$ Department of Electrical and Electronics Engineering, Bilkent University, Bilkent, Ankara, Turkey.

${ }^{*}$ Correspondence to: Ergin Atalar, Ph.D., National Magnetic Resonance Research Center (UMRAM) and Aysel Sabuncu Brain Research Center and Department of Electrical and Electronics Engineering, Bilkent University, 06800, Bilkent, Ankara, Turkey. E-mail: erginatalar@gmail.com

Received 9 August 2017; revised 13 November 2017; accepted 13 November 2017

DOI 10.1002/mrm.27031

Published online 4 December 2017 in Wiley Online Library (wileyonlinelibrary. com).
}

(C) 2017 International Society for Magnetic Resonance in Medicine 400 factors for this technique, especially for a large number of slices, because the SAR and peak $B_{1}$ amplitude increase linearly with the number of slices (4) and the peak RF power increases quadratically with the number of slices. Various techniques have been developed to overcome the limitations of multi-slice RF pulses, such as phase optimization (5), time shifting (6), PINS (7), MultiPINS (8), root flipping (9), optimal control theory (10), and parallel transmission (11-13). All of these RF pulse design methods were developed for conventional linear gradient systems, which provide one-to-one mappings of different slice locations to different frequencies. Therefore, the multi-slice $\mathrm{RF}$ pulses must have either longer durations or higher SARs than single-slice RF pulses.

Alternatively, multiple slice locations can be excited by a single-slice $\mathrm{RF}$ pulse if each slice location is mapped to the same frequency. Therefore, a single-slice RF pulse can also be used for multi-slice excitation without modifications, thereby avoiding increases in the duration, SAR or peak RF power (14). Previously, Parker and Hadley (15) showed that linear gradients can be designed in multiple regions of interest to map multiple regions to the same frequency. In this study, field design methods are presented to dynamically map multiple slice locations in the $\mathrm{z}$ direction to the same resonance frequency, and the resulting field profiles are used to excite multiple slices with a single-band RF pulse. These methods were validated using a z-gradient array in simulations and phantom experiments.

\section{THEORY}

Mapping multiple slice locations to the same frequency requires nonlinear spatial encoding magnetic fields (NSEMs), which are different from conventional linear gradient profiles. Additionally, the slice locations should be dynamically shifted during the sequence to span the entire volume of interest (VOI). Each shifted set of slice locations requires a different N-SEM distribution. To dynamically change the magnetic field distribution, an array of gradient coils is required, and each element should be driven independently with different current weightings to realize a dynamically changing N-SEM profile. In this section, 2 algorithms for designing $\mathrm{N}$ SEMs oscillating in the $\mathrm{z}$ direction for a given set of slice locations and hardware constraints are presented.

\section{Field Design Methods}

Field design methods are formulated to excite $M$ slices with a single-band RF pulse using $N$ independent gradient channels, as shown in Figure 1. For ease of 


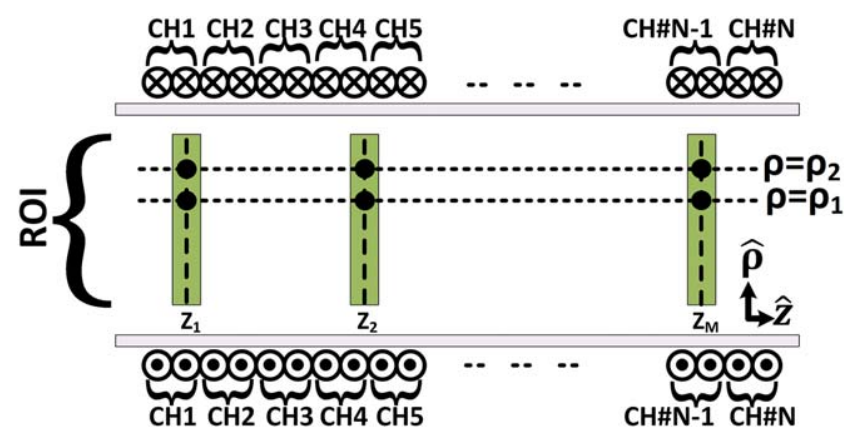

FIG. 1. Schematic illustration of the z-gradient array for multi-slice selection. $N$-channel coil elements with no angular magnetic field variations are used to excite $M$ different slice locations. One or 2 design points can be selected in each slice to determine the weightings of the currents that are applied to each array element to obtain the desired magnetic field distribution.

illustration, each gradient element is wound on the same cylindrical shell with an equal number of sequential turns without separation. The magnetic field generated by each gradient element is angularly invariant. For a given $\rho$ and $z$, the total magnetic field or the first and second derivatives of the magnetic field with respect to the $\mathrm{z}$ direction produced at location $(\rho, z)$ can be written as a linear combination of the contributions from each channel weighted by the current applied to each channel, as shown in Eq. [1]:

$$
B_{z}(\rho, z)=\boldsymbol{P}(\rho, z)^{T} \boldsymbol{I},
$$

where $B_{z}(\rho, z)$ is the total perturbation magnetic field in the $\mathrm{z}$ direction at $(\rho, z)$. The magnetic field is a function of $z$ and the radial distance, $\rho . \boldsymbol{P}(\rho, z)$ is an $N$-element column vector of functions containing single elements $P_{n}$ that represent the magnetic field at $(\rho, z)$ produced by the $n^{\text {th }}$ channel when a unit current is applied. $\boldsymbol{I}$ is an Nx1 column vector containing the currents applied to each channel.

To excite multiple slices with a single-band RF pulse, the magnetic field $B_{z}$ must be designed by optimizing the current weightings of the array elements. The desired magnetic field must map the resonance frequencies of the spins inside the desired slices to the bandwidth of the RF pulse, and all other spins outside the slices should be mapped out of the band of the RF pulse. We propose to use 1 or 2 design points in each slice, referred to as the 1PPS ( 1 point per slice) and 2PPS ( 2 points per slice) methods, respectively. In our approach, the magnetic field distribution at each slice location is similar to a linear gradient profile.

\section{PPS}

In the 1PPS method, a single design point is determined at the center of each slice location, as in Figure 1. At each design point, 3 conditions must be satisfied. First, the magnetic field values at each slice location are equal; therefore, the resonance frequencies of the spins at all design points are forced to be equal. Second, the local gradient strength should be equal at each design point to obtain a constant slice thickness. The gradient strengths at neighboring slice locations are set with alternating polarity. Otherwise, there would be at least 1 other location with the same magnetic field value as the actual desired slice location, which would cause excitation of an additional undesired slice. Even so, alternating polarity of the gradients is necessary but not sufficient condition to avoid undesired excitation between the slices. Third, the second z-derivative of the magnetic fields is set to zero at the design points. Because the magnetic field distribution is angularly invariant and its Laplacian can be assumed to be zero (16), the second derivative with respect to the radial dimension vanishes at the center point. Considering the fact that first derivative with respect the $\rho$ is zero by default because of angular symmetry, zero second derivative helps to extend the designed magnetic field distribution in the slice plane. Moreover, zero second derivative provides locally constant gradient strength in the slice direction. All 3 conditions are formulated as follows:

$$
\begin{aligned}
& B_{z}\left(\rho=0, z_{i}\right)=B_{z}\left(\rho=0, z_{i+1}\right), i=1, \ldots, M-1 \\
& \frac{\partial B_{z}\left(\rho=0, z_{i}\right)}{\partial z}=-\frac{\partial B_{z}\left(\rho=0, z_{i+1}\right)}{\partial z}, i=1, \ldots, M-1 \\
& \frac{\partial^{2} B_{z}\left(\rho=0, z_{i}\right)}{\partial z^{2}}=0, i=1, \ldots, M,
\end{aligned}
$$

where $M$ is the total number of slices and $i$ is the slice index. In Eq. [2], there are a total of $3 M-2$ independent linear equations. Note that even if the magnetic field satisfies these equations, this method does not guarantee a constant magnetic field and a constant magnetic field gradient at all locations in each slice of interest. The performance of this method needs to be investigated through simulations and experiments. Additionally, design point is selected at the center of each slice location throughout the study. However, design point radius can be used as another design parameter without changing the formulation of the method, and some performance parameters depend on the design point radius.

\section{PPS}

Similar to the 1PPS method, in the 2PPS method, the weightings of the currents applied to each gradient element are adjusted to generate magnetic fields that satisfy the following set of equations.

$$
\begin{aligned}
B_{z}\left(\rho_{a}, z_{i}\right) & =B_{z}\left(\rho_{b}, z_{j}\right), i, j=1, \ldots, M \text { and } a, b=1,2, \\
\frac{\partial B_{z}\left(\rho_{a}, z_{i}\right)}{\partial z} & =(-1)^{i-j} \frac{\partial B_{z}\left(\rho_{b}, z_{j}\right)}{\partial z}, i, j=1, \ldots, M \text { and } a, b \\
& =1,2 .
\end{aligned}
$$

Equation [3a] ensures that the magnetic field value is constant at all design points in all slice planes. The second set of equations (Eq. [3b]) has 2 purposes. First, the local gradient strengths at different slice positions are equated up to alternating polarities to ensure a constant slice thickness, as in the 1PPS method. Second, within the same slice, the local gradient strengths at the 2 
design points are equated and assigned the same polarity to extend the constant-slice-thickness region on the slice plane. The first and second sets of equations contribute to the homogeneity of the magnetic field over the transverse plane. Because the magnetic fields are designed at 2 design points in each slice, the profile deviation as a function of radius inside each slice is expected to be lower than in the 1PPS method. The 2PPS method requires $4 \mathrm{M}-2$ equations. This method can be generalized to an arbitrary number $L$ of design points in each slice (LPPS) at the expense of an increased number of linear equations $(2 \times \mathrm{L} \times \mathrm{M}-2)$. Other possible design parameters include the radial coordinates of the design points $\left(\rho=\rho_{1}\right.$ and $\rho_{2}$ ); however, these parameters are considered fixed at $40 \%$ and $95 \%$ of the imaging radius, respectively, throughout this study (see Supporting Fig. S2). Similar to the 1PPS method, the 2PPS method does not ensure a constant slice thickness throughout the entire slice plane. The performance of this method should also be evaluated through simulations and experiments.

Solution of Equations

The current weightings for each channel can be obtained by solving the equations of the 1PPS or 2PPS method. The number of gradient channels is assumed to be greater than the number of equations; therefore, the field equations are always solvable. However, the solution is not unique and has $\mathrm{N}-3 \mathrm{M}+2$ or $\mathrm{N}-4 \mathrm{M}+2$ degrees of freedom for the 1PPS and 2PPS methods, respectively. One way to use the degrees of freedom is to minimize the L2 norm of the current for a given gradient system:

$$
\begin{aligned}
& \min _{I}\|\boldsymbol{I}\|_{2} \text { s.t. } \\
& \boldsymbol{C}^{T} \boldsymbol{I}=0 \\
& \boldsymbol{P}^{\prime}\left(\boldsymbol{\rho}_{a}, z_{i}\right)^{T} \boldsymbol{I}=G_{z} \\
& \|\boldsymbol{I}\|_{\infty} \leq \boldsymbol{I}_{\max },
\end{aligned}
$$

where $\boldsymbol{C}$ is a field constraint matrix with dimensions of either $\mathrm{N} \times(3 \mathrm{M}-2)$ or $\mathrm{N} \times(4 \mathrm{M}-2)$, depending on whether the 1PPS or 2PPS method is used. Each column in $\boldsymbol{C}$ represents a single field equation, determined by inserting Eq. [1] into Eq. [2] for the 1PPS method or into Eq. [3a] for the 2PPS method. $\boldsymbol{P}^{\prime}\left(\rho_{a}, z_{i}\right)$ is the first $\mathrm{z}$ derivative of the previously defined vector $\boldsymbol{P}^{\prime}(\rho, z)$ at any design point, and $G_{z}$ is the target gradient strength at the design points. The final constraint in Eq. [4] ensures that the maximum current that can be applied to the amplifiers, $\mathrm{I}_{\max }$, is not exceeded. Minimization of the L2 norm of the current can also be interpreted as minimization of the total power dissipation if the resistances of the gradient channels are equal.

Equation [4] is a convex quadratic programming problem and can be easily solved using numeric techniques. This problem also has an analytical solution under certain conditions. Because of the field constraints, I must be in the null space of the field constraint matrix $\boldsymbol{C}^{T}$, which is full rank for non-identical slice locations. Another matrix $\boldsymbol{K}$ with dimensions of $9 \times D$ can be constructed: $\boldsymbol{K}=\left[\begin{array}{llll}\boldsymbol{e}_{\boldsymbol{1}} & \boldsymbol{e}_{2} & \ldots & \boldsymbol{e}_{\boldsymbol{D}}\end{array}\right]$, where $\boldsymbol{e}_{j}$ is an orthonormal basis for $\operatorname{null}\left(\boldsymbol{C}^{T}\right)$ and $D$ is the dimensionality of $\operatorname{null}\left(\boldsymbol{C}^{T}\right)$. Therefore, $\boldsymbol{I}$ can be written as $\boldsymbol{I}=\boldsymbol{K} \boldsymbol{\lambda}$, where $\boldsymbol{\lambda}$ is a row vector of length $D$. The optimization problem in Eq. [4] can therefore be converted into another optimization problem with the free parameter $\lambda$ :

$$
\begin{aligned}
& \min _{\lambda}\|\lambda\|_{2} \text { s.t. } \\
& \boldsymbol{P}^{\prime}\left(\rho_{a}, z_{i}\right)^{T} \boldsymbol{K} \lambda=G_{Z} \\
& \|\boldsymbol{K} \lambda\|_{\infty} \leq I_{\max } .
\end{aligned}
$$

If the amplifier limitation constraints are ignored, an analytical solution for $\lambda$ can be found using the pseudoinverse of $\boldsymbol{P}^{\prime}\left(\rho_{a}, z_{i}\right) \boldsymbol{K}$ that results in an optimal current solution to Eq. [4] as follows:

$$
\boldsymbol{I}=G_{z} \frac{\boldsymbol{K} \boldsymbol{K}^{T} \boldsymbol{P}^{\prime}\left(\rho_{a}, z_{i}\right)}{\left\|\boldsymbol{K}^{T} \boldsymbol{P}^{\prime}\left(\rho_{a}, z_{i}\right)\right\|_{2}^{2}}
$$

The analytical minimum norm solution expressed in Eq. [6] is feasible only for $G_{z}$ values less than a certain limiting gradient strength, $G_{l i m}$, which is analytically expressed as

$$
G_{\text {lim }}=I_{\max } \frac{\left\|\boldsymbol{K}^{T} \boldsymbol{P}^{\prime}\left(\rho_{a}, z_{i}\right)\right\|_{2}^{2}}{\left\|\boldsymbol{K} \boldsymbol{K}^{T} \boldsymbol{P}^{\prime}\left(\rho_{a}, z_{i}\right)\right\|_{\infty}}
$$

Otherwise, the amplifier current limitations are exceeded. For higher desired gradient strengths, numerical optimization should be performed. In this study, the analytical solution in Eq. [6] is primarily considered. The minimum norm solution has the advantage that the current vector is only scaled for different gradient strengths for gradient strengths less than $G_{\text {lim }}$. Additionally, this solution enables an analytical definition of a performance measure, $g$, defined as the gradient strength per unit norm current:

$$
g=\frac{G_{Z}}{\|\boldsymbol{I}\|_{2}}=\left\|\boldsymbol{K}^{T} \boldsymbol{P}^{\prime}\left(\rho_{a}, z_{i}\right)\right\|_{2}
$$

Because there is more than a single current flowing in the system, $g$ is defined as the ratio of the gradient strength to the norm of the current; this definition differs slightly from the gradient strength per unit current defined for conventional gradient coils but can nevertheless be intuitively useful. The analytical expression in Eq. [8] is only valid for $G_{z}<G_{\text {lim }}$; when $G_{z}$ is greater than $G_{\text {lim }}, g$ begins to decrease and becomes dependent on $G_{Z}$. The additional degrees of freedom can also be used to maximize $\boldsymbol{P}^{\prime}\left(\rho_{a}, z_{i}\right) \boldsymbol{I}$ under similar conditions for the maximum attainable gradient strength using constraints similar to those in Eq. [4].

\section{METHODS}

\section{Experimental Setup}

For this study, a 9-channel z-gradient coil array was designed, with the topology depicted in Figure 1. The coil array is wound on a plastic cylindrical shell with a diameter of $25 \mathrm{~cm}$ such that each identical array element is directly adjacent to its neighboring element. Each element consists of 36 turns of a $0.85-\mathrm{mm}$ thick copper wire. Because of the continuous winding of the gradient coil 


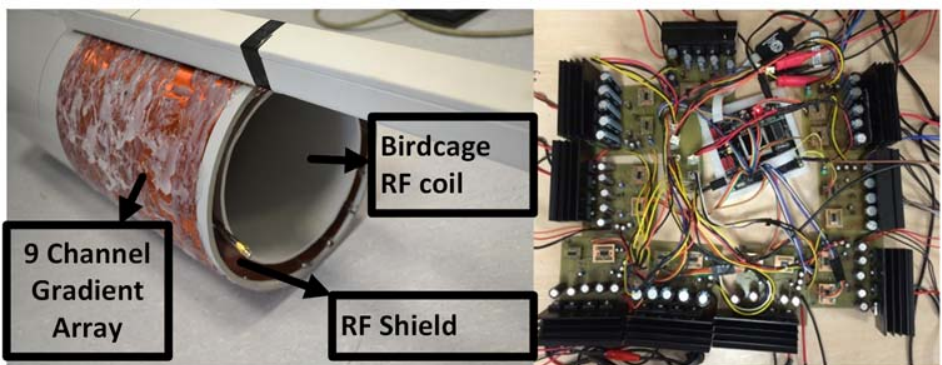

(a)
Gradient Amplifiers

(b)

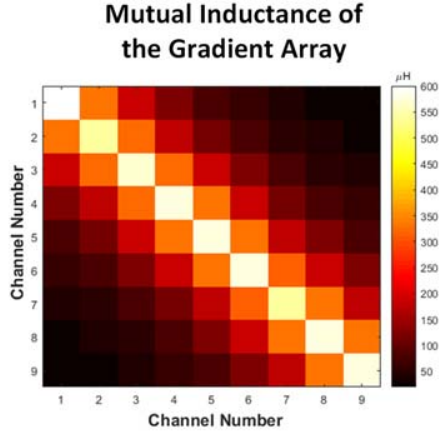

(c)

FIG. 2. Experimental setup. (a) The 9-channel z-gradient coil, the RF coil embedded inside the cylinder and the RF shield. (b) Customdesigned gradient amplifiers for each coil and an FPGA to provide the control signals. (c) The measured mutual inductance matrix, $\boldsymbol{M}$, of the 9-channel z-gradient coil.

and non-zero wire thickness, each element is essentially a helical structure with a pitch angle of $<2^{\circ}$. The feed cables for each channel are designed to be parallel to the $\mathrm{z}$ direction because theoretically, current flow in the $\mathrm{z}$ direction does not contribute to the magnetic field in the $\mathrm{z}$ direction, and force is not induced because of the main magnetic field. Furthermore, a birdcage $\mathrm{Tx} / \mathrm{Rx}$ RF coil with a diameter of $21 \mathrm{~cm}$ and a total length of $22 \mathrm{~cm}$ is placed concentrically with the gradient coil array inside the cylindrical shell. As an RF shield, copper tape is glued to the inner portion of the cylindrical gradient holder, and parts of the shield are removed to form slits in the $\mathrm{z}$ direction to prevent eddy currents on the cylindrical shell because of gradient switching. At each slit, multiple $1 \mathrm{nF}$ capacitors are soldered between the separate parts of the shield to maintain its proper functioning. The gradient coil array, the RF coil and the shield are shown in Figure 2a. All experiments were conducted using a 3T scanner (Magnetom Trio A Tim, Siemens Medical Solutions, Erlangen, Germany) and a sodium-nickel solution phantom (USA Instruments INC, Ohio, USA) with a diameter of $15.3 \mathrm{~cm}$ and $\mathrm{T}_{1}$ of $\sim 110 \mathrm{~ms}$.

To drive each array element independently, 9 customdesigned gradient amplifiers were manufactured. Each amplifier consists of a single h-bridge with maximum voltage and current ratings of $50 \mathrm{~V}$ and $20 \mathrm{~A}$, respectively. Further considerations are necessary for inductive coupling between the array elements. The voltages required for a given current waveform can be calculated as follows:

$$
V_{N \times 1}(t)=\boldsymbol{R}_{N \times N} \cdot \boldsymbol{I}_{N \times 1}(t)+\boldsymbol{M}_{N \times N} \cdot \frac{d}{d t} \boldsymbol{I}_{N \times 1}(t),
$$

where $V(t)$ is the vector of applied voltages for each channel, $\boldsymbol{R}$ is a diagonal matrix representing the resistance of each channel, and $\boldsymbol{M}$ is the mutual coupling matrix, which contains the self-inductances as the diagonal elements and the mutual inductances as the offdiagonal elements.

Pulse-width-modulated (PWM) control signals are provided by the evaluation board of a Xilinx Virtex5 FPGA (XMF5, PLDkit OU). The amplifiers and FPGA board are shown in Figure 2b. A $\mathrm{C}^{++}$-based control interface is used to adjust transistor delays, the reference supply voltage, the arbitrary current waveform for each channel, the resistance of each channel, the mutual coupling matrix for the entire array system and the PWM period. The resistance values for each channel and the mutual inductance matrix were measured using the same experimental setup during the calibration process. The mean and standard deviation of the resistance values for all channel elements are $1.33 \Omega$ and $30 \mathrm{~m} \Omega$. The mutual inductance matrix is shown in Figure 2c; the selfinductance values vary between $540 \mu \mathrm{H}$ and $600 \mu \mathrm{H}$, and the largest mutual inductance between neighboring channels is $340 \mu \mathrm{H}$. After all parameters are specified by the control interface, the FPGA computes the PWM duty cycles using Eq. [9] and the reference voltage.

\section{Coil Field Profiles}

The vector magnetic field profile of each channel was simulated with a $0.2 \mathrm{~mm}$ spatial resolution using the Biot-Savart law. Although a helical coil geometry was considered in the simulations, the field was simulated only on the coronal plane by assuming angular invariance of the three-dimensional distribution because of the very low pitch angle of the coil elements. In the experiments, the $\mathrm{z}$ component of the magnetic field profile of each array element was measured based on the phase difference between a reference coronal gradient-echo (GRE) image and another GRE image with the same echo time and a small blip of current applied to the corresponding array element only during phase encoding. Field maps were obtained with a $1 \mathrm{~mm} \times 1 \mathrm{~mm}$ spatial resolution.

\section{Simulations and Parameters}

The slice locations are defined in terms of slice separation and shift. The slice separation is defined as the distance between the centers of adjacent slice locations. The shift is defined as the offset applied to the $\mathrm{z}=0$ line. A cylindrical volume with a $27 \mathrm{~cm}$ length and $15 \mathrm{~cm}$ diameter (60\% of the coil diameter) is used as the VOI to determine the boundaries of the slice locations.

The 1PPS and 2PPS methods were simulated for several cases with varying slice shifts, slice separations, 


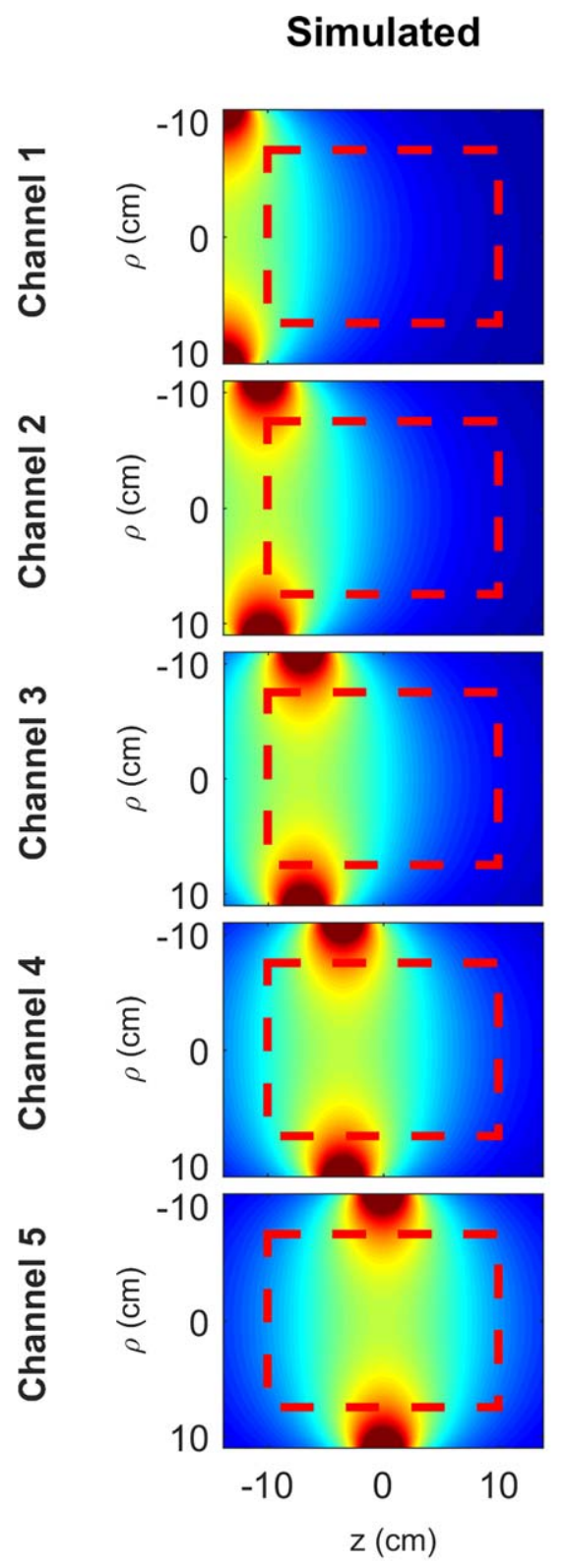

(a)

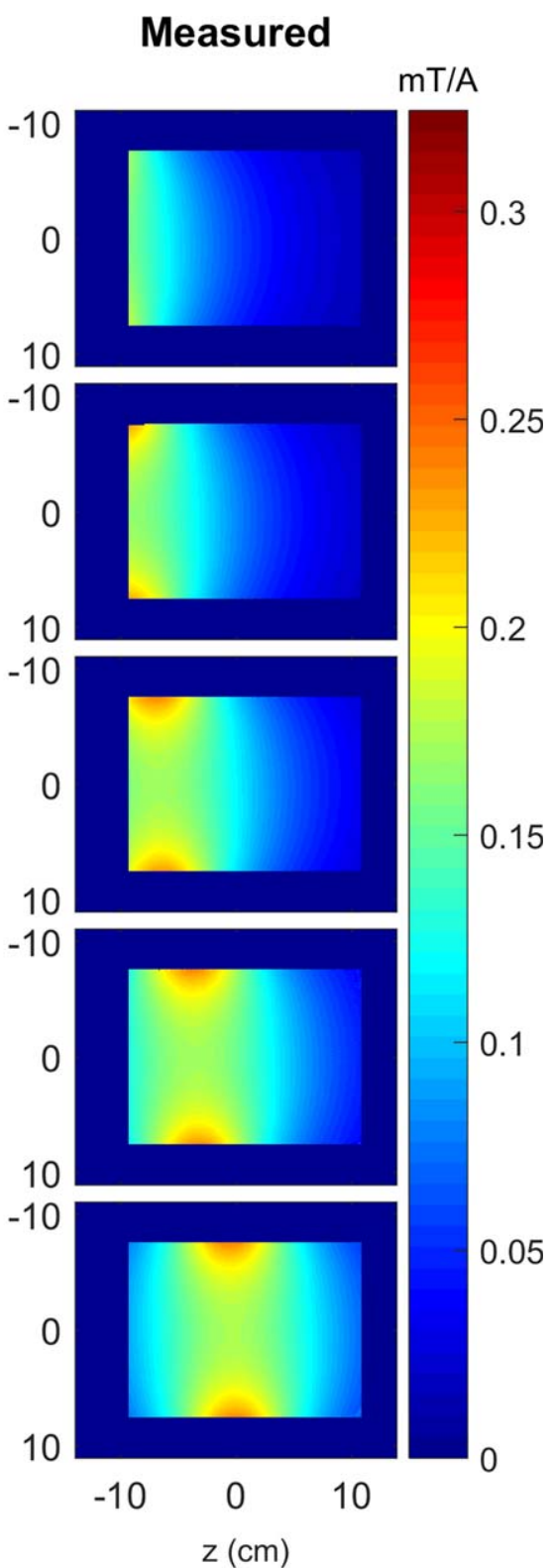

(b) numbers of slices, and design point locations using MATLAB 2016b (The MathWorks, Natick, MA). Because the magnetic fields are designed only at the design points, $\mathrm{z}$ component of the magnetic field and the gradient strength as a function of the transverse radial distance is not constant. Two parameters are adopted as a measure of the slice profile accuracy. First parameter, $\sigma_{\text {center}}$, is the center location variation indicating the standard deviation of the $\mathrm{z}$ coordinates determined by the level set of magnetic field value at the design point as a function of the radius. In other words, $\sigma_{\text {center }}$ quantifies the curvature of the slice shape as a deviation from an ideal central line of the slice. Center location variation is calculated at each slice separately, and $\sigma_{\text {center }}$ is reported as mean of the standard deviation across all slices in a multi-slice scenario. Second parameter is the percentage error of the normalized slice thickness at each radial coordinate on the line determined by the $\mathrm{z}$ coordinate of the slice location is defined with respect to the slice thickness at the design point. To obtain a single performance measure, the root-mean-square error (RMSE) of the normalized slice thickness can be determined by calculating the root-mean-square (RMS) of this value for all points on all slices. Both parameters are not affected by the scaling of the current vector, $I$; therefore, they provide a measure of the magnetic field profile independent of the gradient strength and amplifier specifications. Furthermore, the gradient strength per unit current, $g$, is used to analyze the performance of the design method and the coil geometry independent of the amplifier specifications. Furthermore, the dissipated power, $P_{\text {diss }}$, is calculated as $\boldsymbol{I}^{T} \boldsymbol{R I}$ using the calculated current vector. The maximum amplitude of the $\mathrm{B}$ field inside the VOI, $\mathrm{B}_{\max }$, is calculated by superposing the simulated 3D vector magnetic field distributions of each channel using the weightings specified by $\boldsymbol{I}$. 


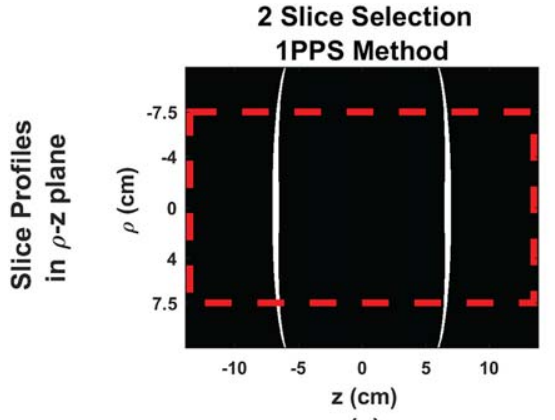

(a)

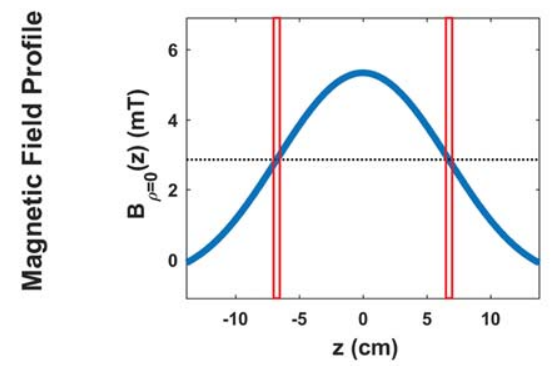

(d)

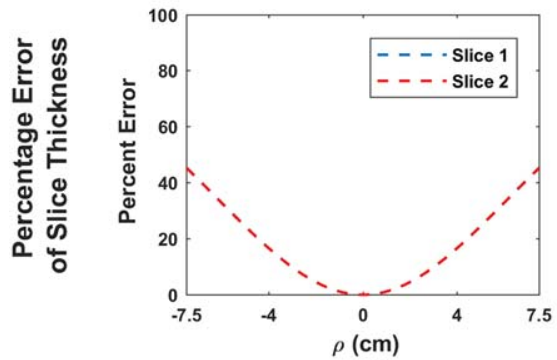

(g)

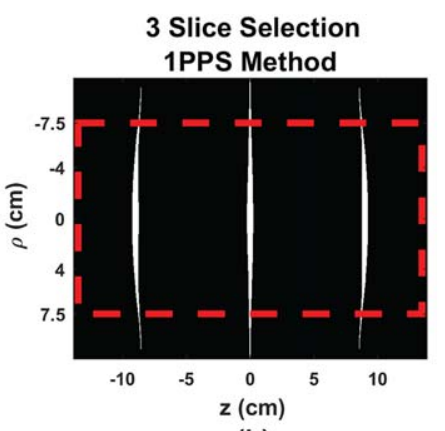

(b)

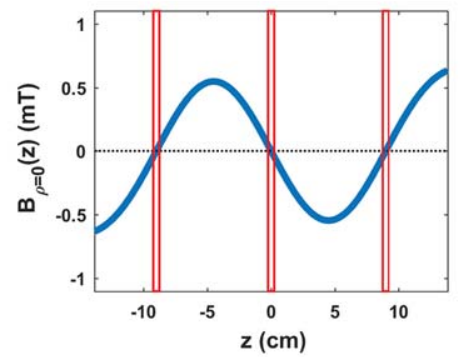

(e)

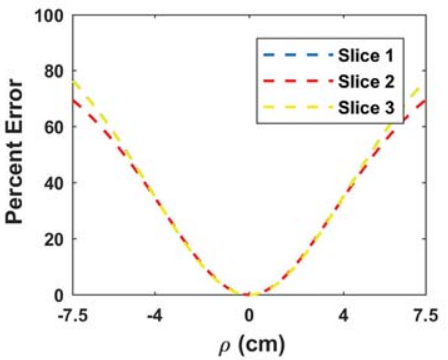

(h)

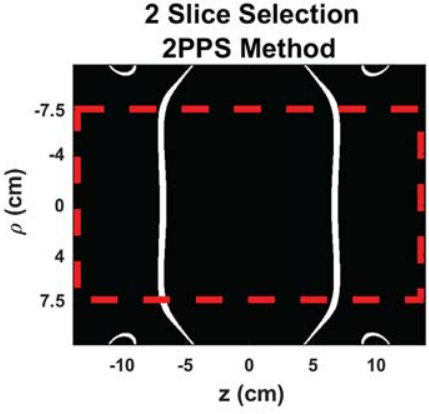

(c)

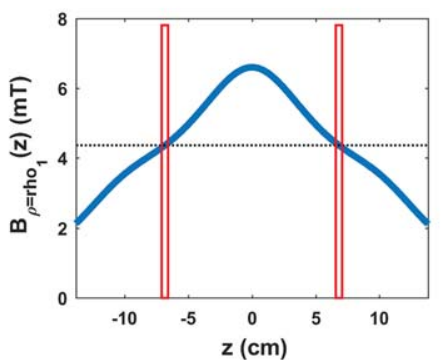

(f)

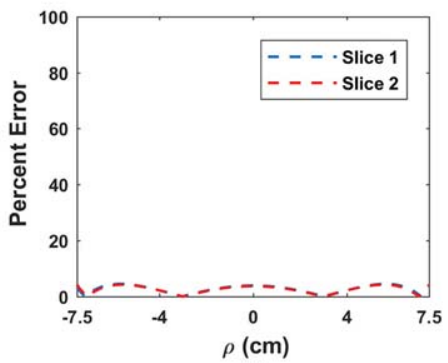

(i)

FIG. 4. Simulated results for 2-slice (left column) and 3-slice selection (middle column) with the 1PPS method and 2-slice selection with the 2PPS method (right column). (a-c) Slice profiles excited by a single band RF pulse. The slice profiles were determined by assuming an ideal RF pulse with a perfectly rectangular slice profile corresponding to a slice thickness of $5 \mathrm{~mm}$ at the design points. The red boxes encompass the entire VOI. (d-f) As examples of spatially oscillating magnetic fields, the one-dimensional magnetic field profiles on the lines $\rho=0$ and $\rho=\rho_{2}$ are shown for the 1PPS and 2PPS methods, respectively. The dashed line corresponds to the RF excitation frequency. The red boxes indicate the multiple slice locations corresponding to the bandwidth of a single-band RF pulse. (g-i) The percentage errors of the slice thickness at all slice locations as a function of radius.

\section{Experiments}

In the experimental validations, measured magnetic field maps were used as inputs to the 1PPS and 2PPS methods. The first and second spatial derivatives of the measured magnetic field distributions are not directly used in the proposed field design methods because any noise in the measured field maps will be amplified by the derivative operation. Instead, the measured magnetic fields are fitted to the simulated magnetic fields along a single line. Fitting is performed for 3 parameters, namely, the amplitude of the magnetic field $(A)$, the spatial shift $(\Delta z)$, and the spatial scaling $(W)$, as follows:

$$
\min _{A, \Delta z, k} \int\left(\tilde{B_{z}}(z)-A B_{z}\left(\frac{z}{W}+\Delta z\right)\right)^{2} d z,
$$

where $B_{z}(z)$ is the simulated magnetic field along a line and $\widetilde{B_{z}}(z)$ is the measured magnetic field along the same line. In the final step, the fitted magnetic field distributions are used to calculate the spatial derivatives of the magnetic fields to prevent noise amplification. Because fitting is performed only to suppress spatial noise, other parameters, such as coil rotation, are neglected.

The current weightings for each channel are calculated using Eq. [6] for a gradient strength of $12.5 \mathrm{mT} / \mathrm{m}$. To obtain a slice thickness of $5 \mathrm{~mm}$, an RF pulse is applied with a Hanning-windowed sinc pulse envelope with a duration of $1.5 \mathrm{~ms}$ and a time-bandwidth product equal to 4 . The gradient amplitudes and the current waveforms, including the refocusing lobe, are specified using the control interface. The slice selection gradient of the original scanner sequence is turned off. RF spoiled 3D GRE sequence was used to validate the design methods. Readout was performed in the $\mathrm{z}$ direction to decrease the spatial resolution in the slice direction to $0.2 \mathrm{~mm}$, whereas the in-plane resolution was $5 \mathrm{~mm}$. The flip angle and pulse repetition time were $40^{\circ}$ and $100 \mathrm{~ms}$, respectively. At the beginning of the experiment, a 


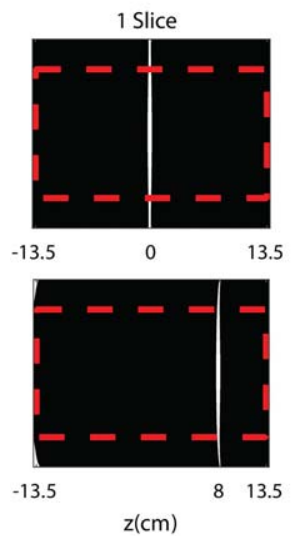

(a)
1PPS Method

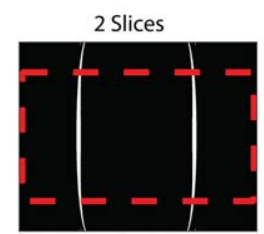

$-6.7$

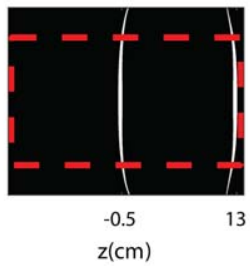

(b)
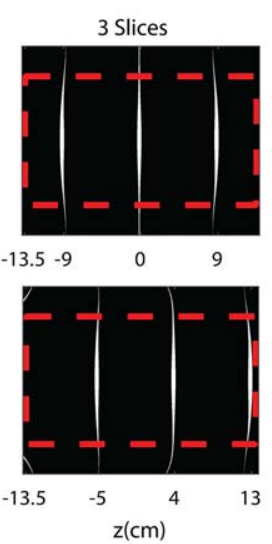

(c)

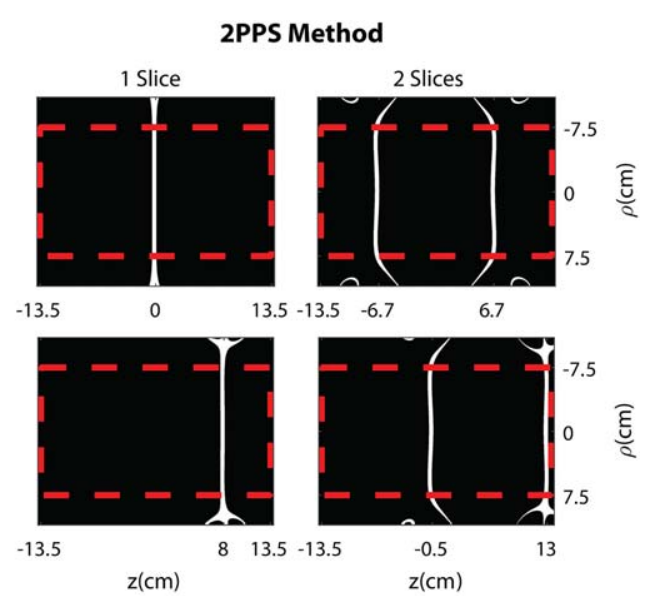

(d)

(e)

FIG. 5. Example slice profiles. (a) 1PPS - 1 slice: (a, row 1) in the center, (a, row 2) shifted by $8 \mathrm{~cm}$. (b) $1 \mathrm{PPS}-2 \mathrm{slices}$ with $13.5 \mathrm{~cm}$ separation: (b, row 1) symmetrically centered and (b, row 2) shifted by $6.4 \mathrm{~cm}$. (c) 1 PPS - 3 slices with $9 \mathrm{~cm}$ separation: (c, row 1) symmetrically centered and (c, row 2) shifted by $4 \mathrm{~cm}$. (d,e) 2PPS - 1 slice (d) and 2 slices (e). The slice shifts and separations for the 2PPS method are the same as those for the 1PPS method with the same number of slices. Slice locations are indicated with labels, and the red dotted window corresponds to the entire VOI.

reference 3D image was obtained without any slice selection. All images were normalized to the reference image to avoid any misleading influence from the Tx/Rx sensitivities of the RF coil on the slice profiles.

\section{RESULTS}

\section{Field Profiles}

In Figure 3, simulated and measured magnetic field profiles per unit current for the first 5 channels are shown. The measurements were performed on a smaller volume determined by the sensitivity of the RF coil. The measurement locations are indicated with red boxes on the simulated profiles. The mean percentage error over all pixels of all channels is $0.6 \%$, and the RMS percentage error is $7 \%$.

\section{Simulations of Slice Profiles}

Because our gradient coil array has 9 elements, a singleband RF pulse can excite a maximum of three or two slices using the 1PPS and 2PPS methods, respectively. Gradient magnetic fields were designed for 2- and 3-slice locations with $13.5 \mathrm{~cm}$ and $9 \mathrm{~cm}$ slice separations using the 1PPS method. The 2PPS method is used to design magnetic fields for 2 slices with a $13.5 \mathrm{~cm}$ separation. (see Fig. 4). The slice separations were selected such that with the shifting of all slices as shown in the following measurements, the entire $27 \mathrm{~cm}$ length of the cylindrical volume could be covered. In Figure 4, the slice locations are centrally symmetric for simplicity, and the slice profiles for multi-slice excitation with a single-band RF pulse are shown. In Figures $4 \mathrm{~d}-4 \mathrm{f}$, the magnetic field profiles along the lines are examples of spatially oscillating N-SEM distributions. The dashed lines and red boxes indicate the center frequency of the RF pulse and the corresponding mapping to the spatial domain for a given slice thickness. Figures $4 \mathrm{a}-4 \mathrm{c}$ show that the slice profiles exhibit a curvature, especially for the 1PPS method. In
Figures $4 \mathrm{~g}-4 \mathrm{i}$, the percentage errors of the slice thickness as a function of $\rho$ are presented. The slice profiles become thinner near the edges. Specifically, for the 1PPS method, the slice thickness is $45 \%$ (2-slice) or $76 \%$ (3slice) lower at the boundary of the VOI than at the center. By contrast, for the 2PPS method, the slice thickness error is $<5 \%$.

The 1PPS and 2PPS methods can similarly be applied for shifted slice locations, as demonstrated in Figure 5. Figures 5a-5c show the results of using the 1PPS method for the excitation of 1,2 , and 3 slices, respectively. Similarly, the use of the 2PPS method to excite a single slice and 2 slices is demonstrated in Figures $5 \mathrm{~d}$ and 5e, respectively. The first row shows centrally symmetric excitation, and the second row shows shifted slice locations with various shifts, as indicated in the figure. Furthermore, the proposed methods are not only valid for multi-slice excitation but also useful for single-slice excitation. Note that multiple-slice locations can be shifted to cover the entire VOI with consecutive multi-slice acquisitions as in Supporting Video S1.

There are possible problems in 3D imaging such as overlaps and gaps between the slices, as slices are shifted to cover the entire VOI because slice thickness and the center location of the slice varies as a function of radius. Total volume coverages of the methods are displayed in Supporting Figures S3a-S3c when each excitation is the 1-slice thickness shifted version of the previous excitation. The bar graph in Figure 3d shows that 1PPS method can excite $74 \%$ and $54 \%$ of the VOI properly for 2 and 3 slices, respectively. The 2PPS method for 2-slice excitation can excite $97 \%$ of the VOI. Remaining volumes can be considered as gaps between the shifted slices because overlapping volumes are $<1 \%$ for all cases.

\section{Effects of Slice Separation and Shift}

The performance of the proposed methods significantly depends on the slice separation and shift. In Figure 6, 


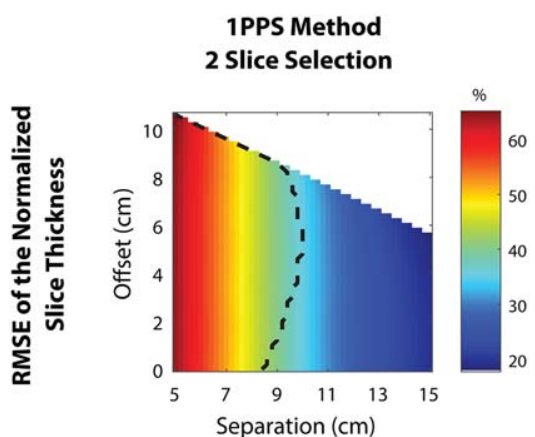

(a)

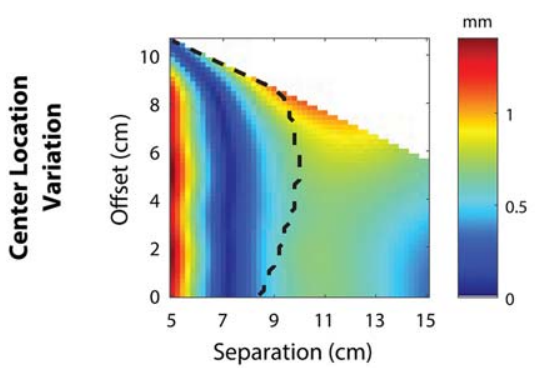

(d)

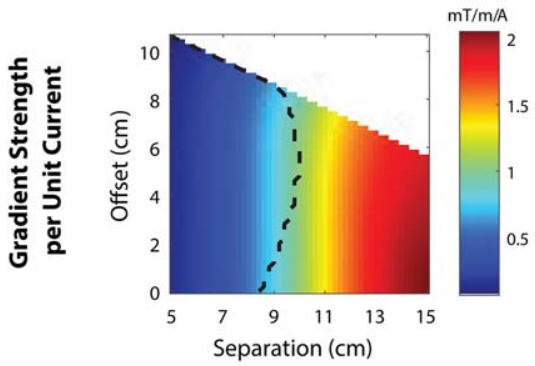

(g)

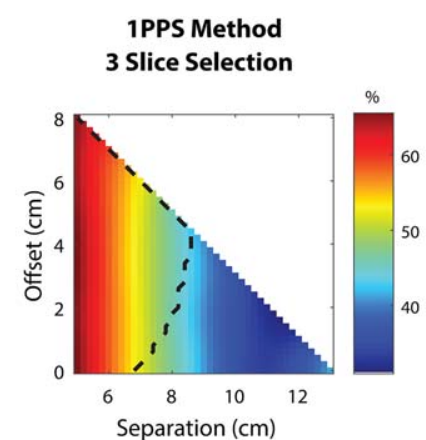

(b)

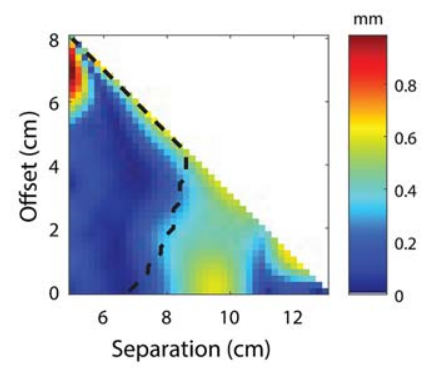

(e)

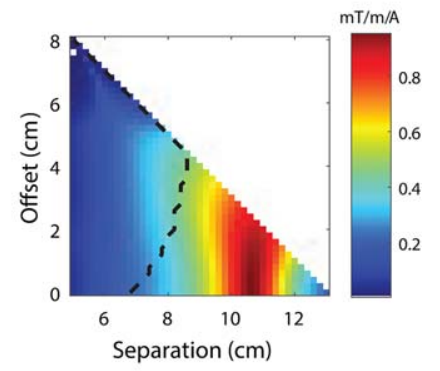

(h)

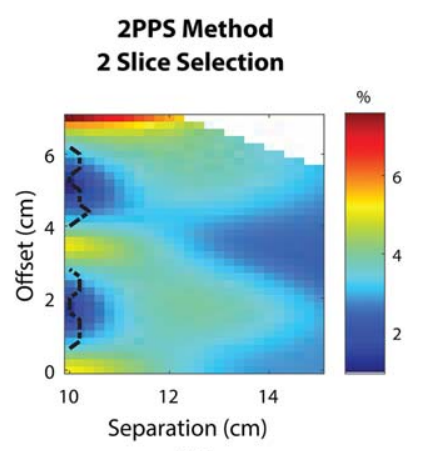

(c)

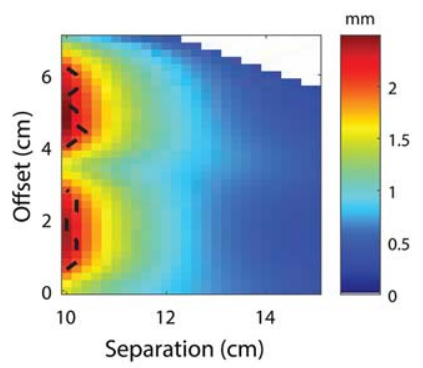

(f)

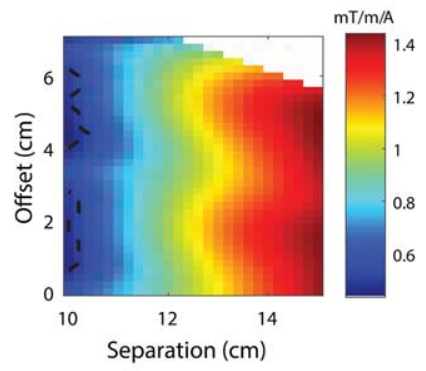

(i)

FIG. 6. Effects of slice separation and shift on system performance. First row: RMSE of the normalized slice thickness over all excited slices. (a) $1 \mathrm{PPS}-2$ slices ( $\min =18 \%$, $\max =65 \%$ ). (b) $1 \mathrm{PPS}-3$ slices $(\min =25 \%$, $\max =66 \%$ ). (c) $2 \mathrm{PPS}-2$ slices ( $\min =1 \%$, $\max =8 \%)$. Second row: center location variation $\left(\sigma_{\text {center }}\right)($ d) 1 PPS -2 slices $(\min =0.0, \max =1.4 \mathrm{~mm})$. (e) 1 PPS -3 slices $(\mathrm{min}=0.0$, $\max =1.0 \mathrm{~mm}$ ). (f) $2 P P S-2$ slices $(\mathrm{min}=0.3, \max =2.5 \mathrm{~mm})$. Third row: gradient strength per unit norm current for the minimum norm solution at the design points (g). (g) 1 PPS -2 slices $(\min =0.1, \max =2.1 \mathrm{mT} / \mathrm{m} / \mathrm{A})$. (h) $1 \mathrm{PPS}-3$ slices $(\mathrm{min}=0.003, \mathrm{max}=1.0 \mathrm{mT} / \mathrm{m} /$ A). (i) $2 P P S-2$ slices $(\min =0.4, \max =1.4 \mathrm{mT} / \mathrm{m} / \mathrm{A})$. The left side of the dashed boundaries corresponds to undesired excitation of a sub-volume inside the VOI.

the simulated RMSE of the normalized slice thickness, center location variation, and the simulated gradient strength per unit norm current are shown for 2- and 3slice excitation with the 1PPS method and 2-slice excitation with the 2PPS method.

The slice thicknesses obtained using the 1PPS method show larger variations in the slice plane compared with the 2PPS method. On the contrary, 1PPS method shows slightly lower center location variation compared to 2PPS method on the average. Additionally, the RMSE of the normalized slice thickness and the gradient strength per unit current primarily depend on the slice separation; the effect of the shift may be ignored for both the 1PPS and 2PPS methods except for smaller slice separations in the case of the 2PPS method. In general, the effect of the shift on the center location variation can still be neglected unless some of the slices are near the edge of the coil. For the 1PPS and 2PPS methods, closer slice locations cause a significant increase in the RMSE of the slice thickness, increased center location variation, and a decrease in the gradient strength per unit norm current. Therefore, the minimum simulated slice separations are $5 \mathrm{~cm}$ for the 1PPS method and $10 \mathrm{~cm}$ for the 2PPS method. For 2-slice excitation with the 1PPS and 2PPS methods, the performances in terms of the RMSE of the slice thickness, the center location variation, and the gradient strength per unit current do not deviate for slice separations $>15 \mathrm{~cm}$. The 1 PPS method results in a higher gradient strength per unit norm current and lower center location variation than the 2PPS method for the excitation of 2 slices with the same separation, at the expense of greater slice thickness variation.

\section{Comparison of the Methods}

The performances of the proposed 1PPS and 2PPS methods are compared in Table 1 in terms of the RMSE of the normalized slice thickness, center location variation $\left(\sigma_{\text {center }}\right), G_{\text {lim }}$, the gradient strength per unit norm current $(g)$, the power dissipation per unit gradient strength, and 
Table 1

Method Comparison

\begin{tabular}{|c|c|c|c|c|c|c|c|c|c|c|}
\hline \multirow[b]{3}{*}{ Slice Number $(N)$} & \multicolumn{6}{|c|}{ 1PPS Method } & \multicolumn{4}{|c|}{ 2PPS Method } \\
\hline & \multicolumn{3}{|c|}{ Shift $=0 \mathrm{~cm}$} & \multicolumn{3}{|c|}{ Shift $=3 \mathrm{~cm}$} & \multicolumn{2}{|c|}{ Shift $=0 \mathrm{~cm}$} & \multicolumn{2}{|c|}{ Shift $=3 \mathrm{~cm}$} \\
\hline & 1 & 2 & 3 & 1 & 2 & 3 & 1 & 2 & 1 & 2 \\
\hline RMSE (\%) & 17 & 23 & 41 & 17 & 23 & 41 & 2 & 3 & 2 & 3 \\
\hline$\sigma_{\text {center }}(\mathrm{mm})$ & 0.0 & 0.5 & 0.5 & 0.1 & 0.6 & 0.4 & 0.0 & 0.6 & 0.1 & 0.6 \\
\hline $\mathrm{G}_{\lim }(\mathrm{mT} / \mathrm{m})$ & 125 & 57 & 19 & 121 & 55 & 20 & 99 & 28 & 95 & 27 \\
\hline $\mathrm{g}(\mathrm{mT} / \mathrm{m} / \mathrm{A})$ & 2.8 & 1.9 & 0.5 & 2.7 & 1.9 & 0.6 & 2.3 & 1.2 & 2.2 & 1.2 \\
\hline $\mathrm{P}_{\text {diss }}(\mathrm{mW})$ & 170 & 360 & 5390 & 180 & 390 & 4170 & 250 & 980 & 260 & 1010 \\
\hline $\mathrm{B}_{\max }(\mathrm{UT})$ & 80 & 136 & 107 & 88 & 156 & 137 & 98 & 244 & 114 & 272 \\
\hline
\end{tabular}

Comparison of the 1PPS and 2PPS methods for different numbers of slices and different shifts in terms of the RMSE of the normalized slice thickness, center location variation $\left(\sigma_{\text {center }}\right), G_{\text {lim }}$, the gradient strength per unit norm current $(g)$, and the power dissipation $\left(P_{\text {diss }}\right)$ and maximum amplitude of the magnetic field $\left(B_{\max }\right)$ for a gradient strength of $1 \mathrm{mT} / \mathrm{m}$.

the maximum magnetic field value per unit gradient strength. The magnetic field distributions are designed for various numbers of simultaneously excited slices for shift values of $0 \mathrm{~cm}$ and $3 \mathrm{~cm}$. A greater number of slices results in decreased $g$, decreased $G_{\text {lim }}$, an increased RMSE of the normalized slice thickness, increased $\sigma_{\text {center}}$, increased dissipated power, and an increased maximum magnetic field. Furthermore, the 1PPS method results in magnetic fields with slightly lower center location variation, higher gradient strength, less dissipated power, and a lower $B_{\max }$ than the magnetic fields generated using the 2PPS method, but the slice thickness error increases with the 1PPS method.

\section{Experimental Validation}

To validate the proposed design methods, the 1PPS and 2PPS methods were applied to both single-slice and multislice excitation with a single-band RF pulse, as shown in Figures 7 and 8, respectively. The 1PPS method is demonstrated for 1, 2, and 3 slices in Figs. 7a-7c. The 2PPS method is demonstrated for 1-slice and 2-slice excitations (a)

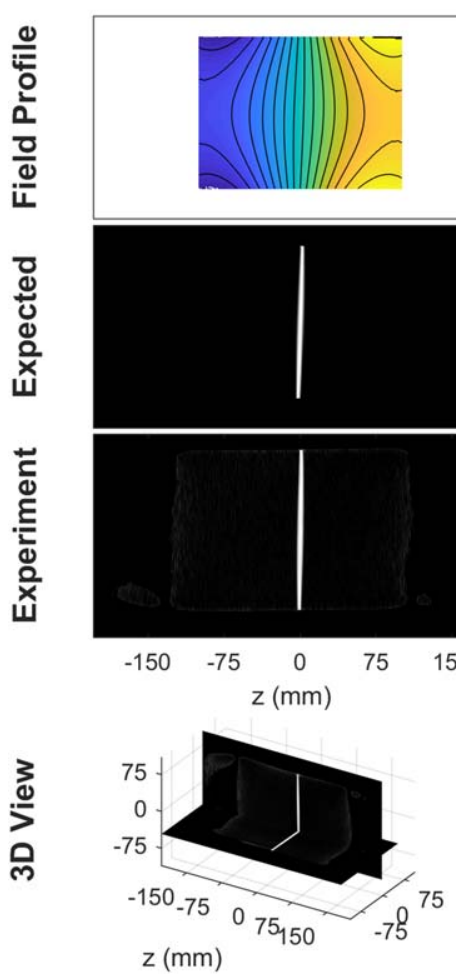

(b)

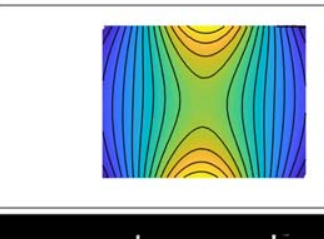

(c) 
(a)
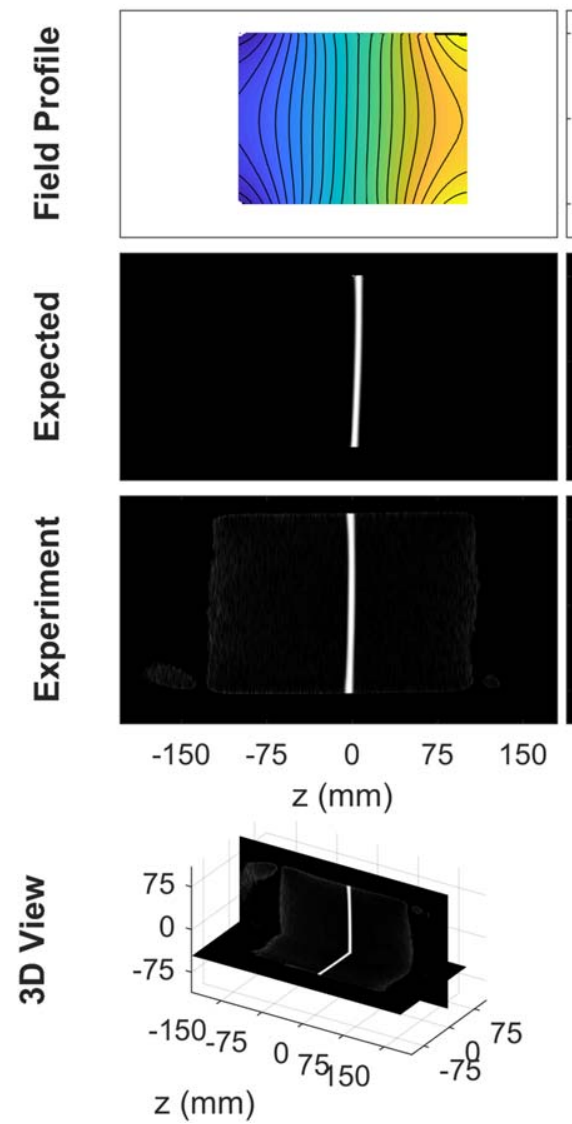

(b)
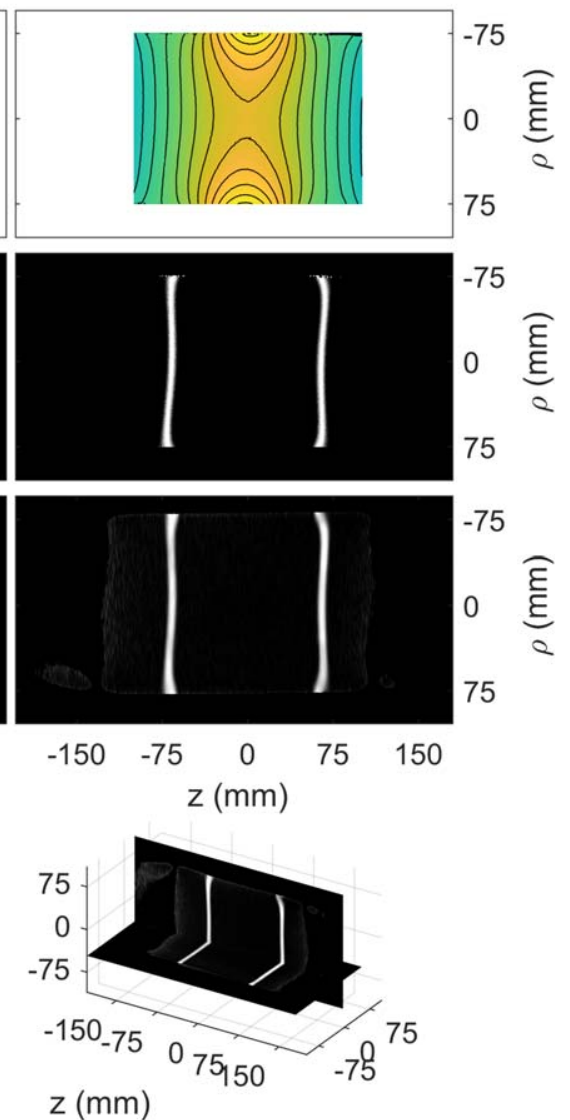

in Figures 8a and 8b. The first row in the figure shows the designed magnetic field for each case and the magnetic field distribution obtained by superposing the field maps of all channels weighted by the optimized current values. The second row shows the expected small-tip-angle slice profiles for a thickness of $5 \mathrm{~mm}$ at the design points under the application of an RF pulse. The third row shows experimental central coronal images to validate the expected slice profiles in the second row. In the last row, 2 example coronal and sagittal images are shown in $3 \mathrm{D}$ views to validate the slice homogeneity in both planes.

Slice profiles for 2-slice excitation with the 2PPS method are plotted in Figure 9. The central coronal image shown in Figure $8 \mathrm{~b}$ is plotted again to present the slice profiles at different radii in Figure. 9a. In Figure 9b, measured and expected slice profiles at the center, at the design points and along arbitrary lines from both the upper and lower half planes are provided. Furthermore, the slice thickness and center location of the slice profile at each radius were calculated by finding the full width at half maximum of the slice profile using cubic interpolation. From the experimental data, the standard deviation of the normalized slice thickness and the center location variation across all radii were calculated to be $11 \%$ and $1 \mathrm{~mm}$, respectively. For the simulations with an ideal coil, the standard deviation of the normalized slice thickness and the center location variation were calculated to be $3 \%$ and $0.6 \mathrm{~mm}$, respectively.
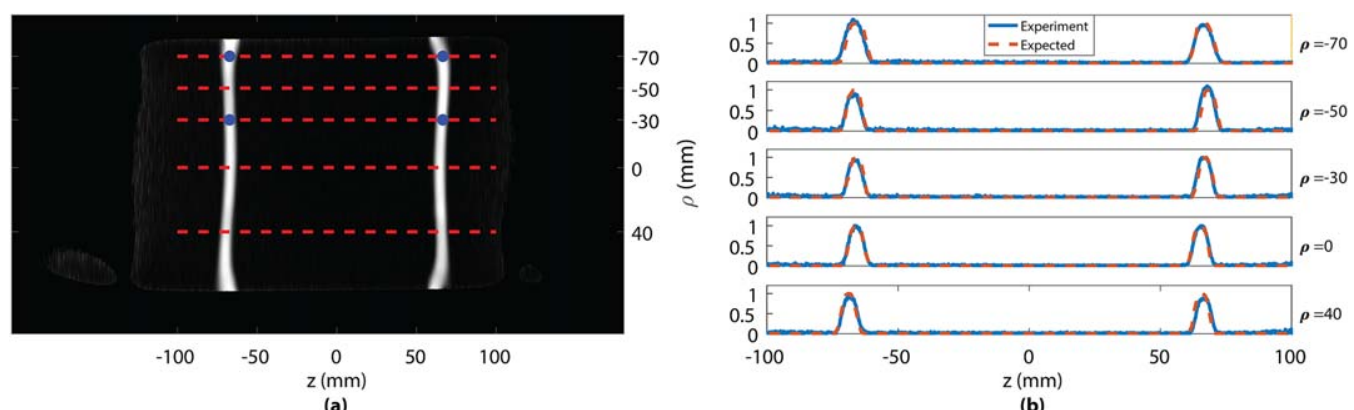

(a)

FIG. 9. (a) Slice profiles measured under excitation using the 2PPS method (blue dots indicate the design points). (b) Line plots of the expected and measured slice profiles at different radii for 2-slice excitation with the 2PPS method. 


\section{DISCUSSION}

\section{RF Pulse Design}

One of the advantages of the proposed methods is that any pulse sequence design algorithm for single-slice excitation, such as the SLR algorithm (17), can be used without any increase in duration, peak power, or SAR as in the case of multi-band RF pulses. Single-slice inversion, refocusing, small-tip-angle, and large-tip-angle RF pulses can be directly used for multi-slice excitation without any modifications because the field profile is designed to produce locally linear gradient fields at the slice locations. Furthermore, techniques for improving single-slice pulse design, such as the VERSE algorithm (18), can be used in combination with the proposed methods for SAR reduction or time optimization.

The number of slices that can be excited with a singleband RF pulse, M, is limited by the number of gradient channels. For the 1PPS method, at least 3M-2 elements are necessary, whereas for the 2PPS method, 4M-2 elements are needed. Another option is to design a magnetic field profile such that multiple, spatially oscillating and wider linear gradient volumes can be obtained using a slightly modified field design method used in this study. Spatially oscillating, wider linear gradient fields can be combined with the multi-band RF pulses to increase the number of slices without increasing the number of channels (19). Moreover, the proposed method is valid only for thin slices $(<5 \mathrm{~mm})$ because the gradient fields are designed only at the center of the slice locations and the slice thickness error increases more rapidly for thicker slices. Spatially oscillating gradient fields in wider regions may be a solution for exciting multiple thicker slices with a single-band RF pulse.

\section{Slice Profile Discrepancy}

The 1PPS and 2PPS methods both have advantages and disadvantages. The slice thickness variation of the 1PPS method is greater compared with the 2PPS method for the same number of slices. The slice profiles become thinner with increasing radius, and the slice thickness variation is less in the center of the slice. In the case of a 3 -slice excitation, only $53.5 \%$ of the overall volume can be covered by regularly shifted slices because of large variation near the edges. To cover the entire VOI, $\sim 3$ times more number of slice shifts are required that offset the advantages of SMS. Instead, this variation can be considered as tolerable for a centrally located region of interest. For example, 1PPS method can excite 95\% of the VOI if diameter of the VOI is reduced to $5 \mathrm{~cm}$. Region of interest does not have to be necessarily centrally located. Off-center design point selection as in Supporting Figure S1 might help to obtain lower thickness variation in the smaller off-center volumes. This drawback of the 1PPS method is compensated for by its superior performance in $G_{\text {lim }}, g, P_{\text {diss }}$ and $B_{\max }$ compared with the 2PPS method. In addition, the 1PPS method can achieve closer slice separations than the 2PPS method, as shown in Figure 6. Therefore, the 1PPS method may be beneficial for the design of ultra-short RF pulses in exchange for increased slice thickness error and/or a reduction in the useful FOV in radial direction. In summary, 1PPS method is weak and not suitable for overall volume imaging; however, it can be preferred for VOIs with smaller radius considering its advantages over 2PPS method.

The simulated slice thickness variation for the 2PPS method is generally $<5 \%$, which is similar to the variation obtained with conventional gradients. Although it is possible to further decrease the slice thickness variation by using more design points per slice (higher $L$ ) and an increased number of channels, the overall system performance decreases dramatically with higher $L$. Slice thickness variations, overlaps, and gaps between shifted slices can cause problems in covering the entire VOI. Accurate reconstruction strategies for overcoming slice discrepancies should be further investigated. For instance, algorithms considering slice profiles similar to SEMAC (20) might be adapted to the proposed method.

The agreement observed here between the expected and measured slice profiles validates the experimental procedure. There are possible reasons for the higher slice thickness variations in the experiments compared with the simulations. First, flip angle was selected as $40^{\circ}$ for a phantom. Steady-state longitudinal magnetization before the RF pulse decreases to $86 \%$ of its initial value considering the $\mathrm{T}_{1}$ of the phantom and pulse repetition time of the sequence; therefore, saturation effects can slightly increase the apparent slice thickness. Other possible reason is the imperfections in the home-built gradient coil array. In both the 1PPS and 2PPS methods, angular symmetry of the coils is assumed in the equations; however, the measured magnetic field maps of the manufactured coils are slightly tilted because of manufacturing imperfections. Consequently, the thickness variations in the lower half plane were not well controlled in these experiments because both design points were chosen to lie in the upper half plane. However, such thickness variations might still be acceptable.

\section{Dynamic Adaptation of the Field Profile}

Independent control of each array element enables the dynamic adaptation of the field profile. The 1PPS and 2PPS methods lead to $3 \mathrm{M}-2$ and $4 \mathrm{M}-2$ field constraints, respectively. The remaining degrees of freedom are used to minimize the norm of the current which also minimizes the $P_{\text {diss }}$ in case of identical resistances. However, they can also be used to reduce the $B_{\max }$, or the slice thickness variation or to increase the $G_{\max }$. A lower $B_{\max }$ may result in less stored inductive energy and might better respect peripheral nerve stimulation limitations because of lower E fields and potentially higher slew rates. Additionally, the design point locations, which are important parameters affecting the slice thickness variation, center location variation, and $g$, can be independently optimized for each slice location and VOI (see Supporting Figs. S1 and S2). Moreover, combination of constraints for the equal magnetic fields, first and second derivative of the fields can be used separately for different design points that can use the tradeoff between slice thickness variation, center location variation, and $g$ according to design specifications. Although such optimizations have not been studied in 
detail, a higher number of degrees of freedom is promising for greater adaptability of the possible field profiles.

Unlike for a coil array, for conventional linear gradient coils, the specifications for the VOI, linearity error and gradient strength per unit current are determined at the beginning of design. The field profile cannot be changed after coil manufacture. However, there is a tradeoff between the VOI, the linearity error and the gradient strength specifications that may vary dramatically with different sequences, different target organs, or other factors. Furthermore, another advantage of dynamic adaptation of the field profile is to extend the useful VOI of the coil. For instance, if a conventional gradient coil were to be designed with the same physical dimensions and the same aspect ratio, reasonable homogeneity could be achieved in a volume of $15-20 \mathrm{~cm}$ in length in the $\mathrm{z}$ direction, whereas our coil array can excite slices even at the edge of the coil in a $27 \times 15 \mathrm{~cm}$ cylindrical VOI.

\section{Limitations}

In addition to slice thickness variations, another significant limitation of our methods is the dramatic decrease in performance for smaller slice separations. Although the 1PPS method can achieve the excitation of closer slices than the 2PPS method, its performance decreases. The radius of the VOI is an important parameter affecting both the minimum slice separation and the tolerable slice thickness variation. Perhaps, a different hardware geometry would be required to further reduce the slice separation while maintaining performance. The target field method (21) might provide intuition for the required current density on the coil for a target magnetic field distribution specified on a cylindrical surface. For the angularly symmetric coils, current density and the magnetic field profile on a cylindrical surface are related by the spatial Fourier transform in the z-direction (21). Application of our method for smaller slice separations forces desired field to contain higher spatial frequency content which implies higher spatial frequency content for the current density on the coil. Such a current distribution requires shorter array elements to realize rapidly varying, high frequency current distribution. Such a rapid variation in the current density also decreases the strength of the generated field inside the VOI; therefore, current requirement of the amplifiers would be increased significantly. Excitation of the closer slices can be considered as the main weakness of the study and requires further investigation.

Slew rate is not considered in this paper; however, it is very significant for practical applications. Because of hardware limitations, a simplistic model is presented in Eq. [8] for calculating the required voltages of the amplifiers. However, it is difficult to report a single maximum slew rate value because the current in each channel affects the other channels via mutual coupling. Similarly, there is no single slew rate limitation in terms of peripheral nerve stimulation because the E-field distributions of the coil elements are superposed with dynamically changing current weightings. However, this effect should be analyzed in future studies of gradient coil arrays for human applications.
There are also practical limitations regarding the extension of this work to clinical whole-body coil arrays. First, the current study needs to be extended to the excitation of slices in arbitrary orientations. This might be achieved with a more generalized array of coils. The angular symmetry assumption will fail for the generalized array of coils; therefore, more general formulations will be required. The generalized array of coils might also provide lower slice thickness variation and center location variation because of the increased degree of freedom in the system in all directions. Moreover, the array elements are not shielded. Eddy current effects are neglected in this study because of the relatively small coil radius compared to the magnet radius. For largerradius applications, either each element must be selfshielded (22) or a shield can be designed as an array of coils. The power requirements for a gradient coil increase dramatically with increasing radius (23); therefore, more powerful amplifiers will be required.

\section{Other Applications of N-SEMs}

Although the proposed methods create locally linear gradient fields, these fields can also be regarded as N-SEMs in general. N-SEMs have proven to be useful in many aspects of both the reception (24-31) and excitation (32-38) phases of an imaging sequence. Therefore, increased hardware complexity is useful for many other purposes in addition to multi-slice excitation with a single-band RF pulse. If some portion of the VOI except the desired slice locations are excited as mentioned in Figure 6, the combination of parallel excitation with NSEMs can suppress the additional undesired excitations (39). Additionally, several groups have designed gradient coil arrays, such as multi-coils (40) and matrix gradient coils (22), with a larger number of channels, thereby proving the feasibility of the necessary hardware. A larger number of gradient coils can be used for higherorder shimming (41) to eliminate the need for separate shim coils.

In this study, 2 design methods are introduced for exciting multiple slices in the $\mathrm{z}$ direction with a singleband RF pulse using a 9-channel gradient array system. The feasibility of the proposed methods is validated through simulations and phantom experiments, and performance analyses are provided.

\section{ACKNOWLEDGMENTS}

The authors thank Sercan Aydoğmuş and Hamed Mohammadi for designing the control interface for the gradient array system and Mustafa Can Delikanlı for assisting with the production of the gradient coils.

\section{REFERENCES}

1. Gagoski BA, Bilgic B, Eichner C, Bhat H, Grant PE, Wald LL, Setsompop K. RARE/turbo spin echo imaging with simultaneous multislice Wave-CAIPI. Magn Reson Med 2015;73:929-938.

2. Moeller S, Yacoub E, Olman CA, Auerbach E, Strupp J, Harel N, Ugurbil K. Multiband multislice GE-EPI at 7 tesla, with 16 -fold acceleration using partial parallel imaging with application to high spatial and temporal whole-brain fMRI. Magn Reson Med 2010;63:11441153. 
3. Setsompop K, Gagoski BA, Polimeni JR, Witzel T, Wedeen VJ, Wald LL. Blipped-controlled aliasing in parallel imaging for simultaneous multislice echo planar imaging with reduced g-factor penalty. Magn Reson Med 2012;67:1210-1224.

4. Barth M, Breuer F, Koopmans PJ, Norris DG, Poser BA. Simultaneous multislice (SMS) imaging techniques. Magn Reson Med 2016;75:63-81.

5. Sbrizzi A, Poser BA, Tse DH, Hoogduin H, Luijten PR, Berg CA. RF peak power reduction in CAIPIRINHA excitation by interslice phase optimization. NMR Biomed 2015;28:1393-1401.

6. Auerbach EJ, Xu J, Yacoub E, Moeller S, Uğurbil K. Multiband accelerated spin-echo echo planar imaging with reduced peak RF power using time-shifted RF pulses. Magn Reson Med 2013;69:1261-1267.

7. Norris DG, Koopmans PJ, Boyacioğlu R, Barth M. Power independent of number of slices (PINS) radiofrequency pulses for low-power simultaneous multislice excitation. Magn Reson Med 2011;66:1234-1240.

8. Eichner C, Wald LL, Setsompop K. A low power radiofrequency pulse for simultaneous multislice excitation and refocusing. Magn Reson Med 2014;72:949-958.

9. Sharma A, Lustig M, Grissom WA. Root-flipped multiband refocusing pulses. Magn Reson Med 2016;75:227-237.

10. Aigner CS, Clason C, Rund A, Stollberger R. Efficient high-resolution RF pulse design applied to simultaneous multi-slice excitation. J Magn Reson 2016;263:33-44.

11. Poser BA, Anderson RJ, Guérin B, Setsompop K, Deng W, Mareyam A, Serano P, Wald LL, Stenger VA. Simultaneous multislice excitation by parallel transmission. Magn Reson Med 2014;71:1416-1427.

12. Wu X, Schmitter S, Auerbach EJ, Moeller S, Uğurbil K, de Moortele V. Simultaneous multislice multiband parallel radiofrequency excitation with independent slice-specific transmit B1 homogenization. Magn Reson Med 2013;70:630-638.

13. Guérin B, Setsompop K, Ye H, Poser BA, Stenger AV, Wald LL. Design of parallel transmission pulses for simultaneous multislice with explicit control for peak power and local specific absorption rate. Magn Reson Med 2015;73:1946-1953.

14. Ertan K, Taraghinia S, Sadeghi A, Atalar E. A z-gradient array for spatially oscillating magnetic fields in multi-slice excitation. In Proceedings of the 33rd Annual Meeting of ESMRMB, Vienna, Austria, 2016. Abstract 81.

15. Parker DL, Hadley JR. Multiple-region gradient arrays for extended field of view, increased performance, and reduced nerve stimulation in magnetic resonance imaging. Magn Reson Med 2006;56:1251-1260.

16. Bernstein MA, Zhou XJ, Polzin JA, King KF, Ganin A, Pelc NJ, Glover GH. Concomitant gradient terms in phase contrast MR: analysis and correction. Magn Reson Med 1998;39:300-308.

17. Pauly J, Le Roux P, Nishimura D, Macovski A. Parameter relations for the Shinnar-Le Roux selective excitation pulse design algorithm (NMR imaging). IEEE Trans Med Imaging 1991;10:53-65.

18. Conolly S, Nishimura D, Macovski A, Glover G. Variable-rate selective excitation. J Magn Reson 1988;78:440-458.

19. Ertan K, Taraghinia S, Mohammadi H, Atalar E. Gradient field design for RF excitation in simultaneous multi-slice and simultaneous multi-slab imaging by using a z-gradient array. In Proceedings of the 25th Annual Meeting of ISMRM, Honolulu, Hawaii, USA, 2017. Abstract 423.

20. Lu W, Pauly KB, Gold GE, Pauly JM, Hargreaves BA. SEMAC: slice encoding for metal artifact correction in MRI. Magn Reson Med 2009; 62:66-76.

21. Turner R. A target field approach to optimal coil design. J Phys D Appl Phys 1986;19:L147-L151.

22. Littin S, Jia F, Layton KJ, Kroboth S, Yu H, Hennig J, Zaitsev M. Development and implementation of an 84-channel matrix gradient coil. Magn Reson Med 2018;79:1181-1191.

23. Bowtell R, Mansfield P. Gradient coil design using active magnetic screening. Magn Reson Med 1991;17:15-21.

24. Stockmann JP, Ciris PA, Galiana G, Tam L, Constable RT. O-space imaging: highly efficient parallel imaging using second-order nonlinear fields as encoding gradients with no phase encoding. Magn Reson Med 2010;64:447-456.

25. Tam LK, Stockmann JP, Galiana G, Constable RT. Null space imaging: nonlinear magnetic encoding fields designed complementary to receiver coil sensitivities for improved acceleration in parallel imaging. Magn Reson Med 2012;68:1166-1175.

26. Weber H, Schultz G, Gallichan D, Hennig J, Zaitsev M. Local field of view imaging for alias-free undersampling with nonlinear spatial encoding magnetic fields. Magn Reson Med 2014;71:1002-1014.
27. Layton KJ, Gallichan D, Testud F, Cocosco CA, Welz AM, Barmet C, Pruessmann KP, Hennig J, Zaitsev M. Single shot trajectory design for region-specific imaging using linear and nonlinear magnetic encoding fields. Magn Reson Med 2013;70:684-696.

28. Wiesler DG, Wen H, Wolff SD, Balaban RS. Reduction of field of view in MRI using a surface-spoiling local gradient insert. J Magn Reson Imaging 1998;8:981-988.

29. Hennig J, Welz AM, Schultz G, Korvink J, Liu Z, Speck O, Zaitsev M. Parallel imaging in non-bijective, curvilinear magnetic field gradients: a concept study. MAGMA 2008;21:5-14.

30. Gallichan D, Cocosco CA, Dewdney A, Schultz G, Welz A, Hennig J, Zaitsev M. Simultaneously driven linear and nonlinear spatial encoding fields in MRI. Magn Reson Med 2011;65:702-714.

31. Schultz G, Ullmann P, Lehr H, Welz AM, Hennig J, Zaitsev M. Reconstruction of MRI data encoded with arbitrarily shaped, curvilinear, nonbijective magnetic fields. Magn Reson Med 2010;64:13901403.

32. Börnert P, Schäffter T. Curved slice imaging. Magn Reson Med 1996; 36:932-939.

33. Weber H, Gallichan D, Schultz G, Cocosco CA, Littin S, Reichardt W, Welz A, Witschey W, Hennig J, Zaitsev M. Excitation and geometrically matched local encoding of curved slices. Magn Reson Med 2013;69:1317-1325

34. Weber H, Haas M, Kokorin D, Gallichan D, Hennig J, Zaitsev M. Local shape adaptation for curved slice selection. Magn Reson Med 2014; 72:112-123.

35. Ma C, Xu D, King KF, Liang ZP. Reduced field-of-view excitation using second-order gradients and spatial-spectral radiofrequency pulses. Magn Reson Med 2013;69:503-508.

36. Witschey WR, Cocosco CA, Gallichan D, Schultz G, Weber H, Welz A, Hennig J, Zaitsev M. Localization by nonlinear phase preparation and k-space trajectory design. Magn Reson Med 2012;67:1620-1632.

37. Islam H, Glover GH. Reduced field of view imaging using a static second-order gradient for functional MRI applications. Magn Reson Med 2016;75:817-822.

38. Ertan K, Atalar E. Simultaneous use of linear and nonlinear gradients for B1 + inhomogeneity correction. NMR Biomed 2017;30:e3742.

39. Haas M, Ullmann P, Schneider J, Post H, Ruhm W, Hennig J, Zaitsev M. PexLoc-parallel excitation using local encoding magnetic fields with nonlinear and nonbijective spatial profiles. Magn Reson Med 2013;70:1220-1228.

40. Juchem C, Rudrapatna SU, Nixon TW, de Graaf RA. Dynamic multicoil technique (DYNAMITE) shimming for echo-planar imaging of the human brain at 7 Tesla. Neuroimage 2015;105:462-472.

41. Juchem C, Nixon TW, McIntyre S, Boer VO, Rothman DL, de Graaf RA. Dynamic multi-coil shimming of the human brain at 7T. J Magn Reson 2011;212:280-288.

\section{SUPPORTING INFORMATION}

Additional Supporting Information may be found in the online version of this article.

Fig. S1. Performance evaluation of design point selection in the 1PPS method for excitation of 2 and 3 slices with a separation of $9 \mathrm{~cm}$ including both centrally symmetric and $3 \mathrm{~cm}$ shifted slices. (a) RMSE of the normalized slice thickness (b) center location variation ( $\left.\sigma_{\text {center }}\right)$ (c) gradient strength per unit norm current $(\mathrm{g})$ as a function of design point radius are plotted.

Fig. S2. Performance evaluation of design point selection in the 2PPS method for excitation of 2 slices with a separation of $13.5 \mathrm{~cm}$. (a) RMSE of the normalized slice thickness for symmetric placement of slices around the center. (b) RMSE of the normalized slice thickness for slice locations as shifted $+3 \mathrm{~cm}$ according to the previous case. (c) Center location variation for symmetric slices. (d) Center location variation for shifted slices. (e) Gradient strength per unit current norm for symmetric slices. (f) Gradient strength per unit current norm for shifted slices. Red dot indicates the choice of the design points used in the entire study.

Fig. S3. Representation of total excitation when the set of shifted slices are excited evenly to cover the entire VOI. (a) 1PPS, 2 slice. (b) 1PPS, 3 slice. (c) 2PPS, 2 slice. Blue rectangle represents the VOI. Volume is classified into 3 sub-volumes such as (black) gaps that are not excited by any of the excitations, (green) properly excited once, (red) overlaps that are excited more than once during the set of shifted slice excitations. (d) Bar graph representation for percentages of sub-volume for $(a-c)$. 2D images are integrated to calculate the volume fractions in 3D.

Video S1. Demonstration of VOI coverage as slice locations are shifted. 